Article

\title{
Structural Characterization and Antioxidant Activities of Polysaccharides Extracted from the Pulp of Elaeagnus angustifolia L.
}

\section{Qingqing Chen ${ }^{1, \dagger}$, Juncheng Chen ${ }^{2, \dagger}$, Hongtao Du ${ }^{2}, \mathrm{Qi} \mathrm{Li}^{3}$, Jun Chen ${ }^{3}$, Gechao Zhang ${ }^{2}$, Hong Liu ${ }^{1, *}$ and Junru Wang ${ }^{2, *}$}

1 College of Science, Shihezi University, Shihezi 832003, XinJiang, China;

E-Mail: chenqingqing1027@gmail.com

2 College of Science, Northwest A\&F University, Yangling 712100, Shaanxi, China;

E-Mails: chenjc@gmail.com (J.C.); duht@nwsuaf.edu.cn (H.D.); zhanggc@gmail.com (G.Z.)

3 Department of Biology, Yangling Hi-Tech Middle School, Yangling 712100, Shaanxi, China; E-Mails: liqi@gmail.com (Q.L.); chenj@gmail.com (J.C.)

$\dagger$ These authors contributed equally to this work.

* Authors to whom correspondence should be addressed;

E-Mails: lhong@shzu.edu.cn (H.L.); wangjunru@nwsuaf.edu.cn (J.W.);

Tel.: +86-180-9758-6569 (H.L.); +86-151-0927-3921 (J.W.);

Fax: +86-993-205-5655 (H.L.); +86-29-8709-2829 (J.W.).

Received: 2 April 2014; in revised form: 12 May 2014 / Accepted: 28 May 2014 /

Published: 26 June 2014

\begin{abstract}
In this study, two polysaccharides (Elaeagnus angustifolia L. polysaccharide-1 (PEA-1) and PEA-2) were prepared from Elaeagnus angustifolia L. Then, the preliminary structure and antioxidant activities of all the samples were investigated. The results showed that the average molecular weights for PEA-1 and PEA-2 were 9113 and 5020 Da, respectively. And, PEA-1 was mainly composed of rhamnose, xylose, mannose, glucose, and galactose, respectively. The components of PEA-2 were rhamnose, mannose, glucose, and galactose, respectively. Moreover, the Antioxidant assays demonstrated that PEA-1 possessed of strong free radicals scavenging activity and hydroxyl radicals scavenging activities, suggesting that PEA-1 could potentially be used as natural antioxidant.
\end{abstract}


Keywords: Elaeagnus angustifolia L.; polysaccharides; isolation; structural characterization; antioxidant activity

\section{Introduction}

Elaeagnus angustifolia L., namely shazao in China, contains multiple chemical constituents, including proteins, flavonoids, amino acids, polysaccharides, and inorganic elements, etc. [1]. And, it has a variety of medicinal uses. Such as it is employed in the treatment of nausea, vomiting, jaundice, asthma, and flatulence [2-4]. The pulp of Elaeagnus angustifolia L. have been used to treat amoebic dysentery. Antibacterial agents, such as epigallocatechin from the bark of E. glabra, were identified [5]. The flavonoid of Elaeagnus angustifolia L. showed significant antinociceptive activity and muscle relaxant activity $[6,7]$.

The polysaccharides, as an important component of Elaeagnus angustifolia L., have anti-radiation activity, immunoregulatory activity, and antioxidant activity [8,9]. Our group reported that the crude polysaccharide could reinforce immune function of experimental mice [10]

Based on the above information, the aim of this work is to prepare pure polysaccharides of Elaeagnus angustifolia L. fruits, and investigate the preliminary characterization, antioxidants, in vitro.

\section{Results and Discussion}

\subsection{Isolation and Purification of Polysaccharides}

The crude polysaccharide was extracted from Elaeagnus angustifolia L. pulp. The crude polysaccharide was isolated three fractions using a deae cellulose 52 (DE-52) column. The neutral polysaccharide fraction, eluted with water, was further fractionated using a G-100 column, and a major extracellular polysaccharides PEA-1 and PEA-2 was obtained (Figure 1).

Figure 1. Extraction and isolation scheme.

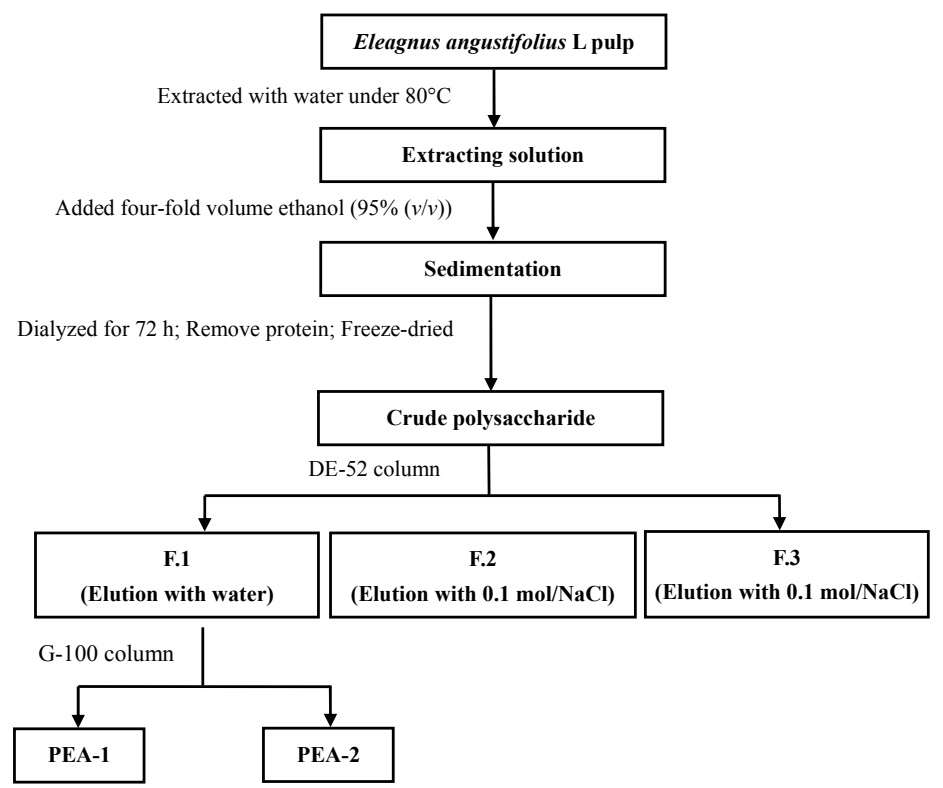




\subsection{Preliminary Characterization of Elaeagnus angustifolia L. Polysaccharide (PEA)}

\subsubsection{Molecular Weight of PEA}

HPLC system was used to determine the homogeneity and molecular weight of PEA-1 and PEA-2. As shown in Figure 2, the HPLC profile of each purified fraction had a single and symmetrical narrow peak, which indicated that they were homogeneous polysaccharides. In addition, the average molecular weights of PEA-1 and PEA-2 were estimated to be 9113 and $5020 \mathrm{Da}$, respectively.

Figure 2. HPGPC chromatograms of (a) PEA-1 and (b) PEA-2 of molecular weigths.
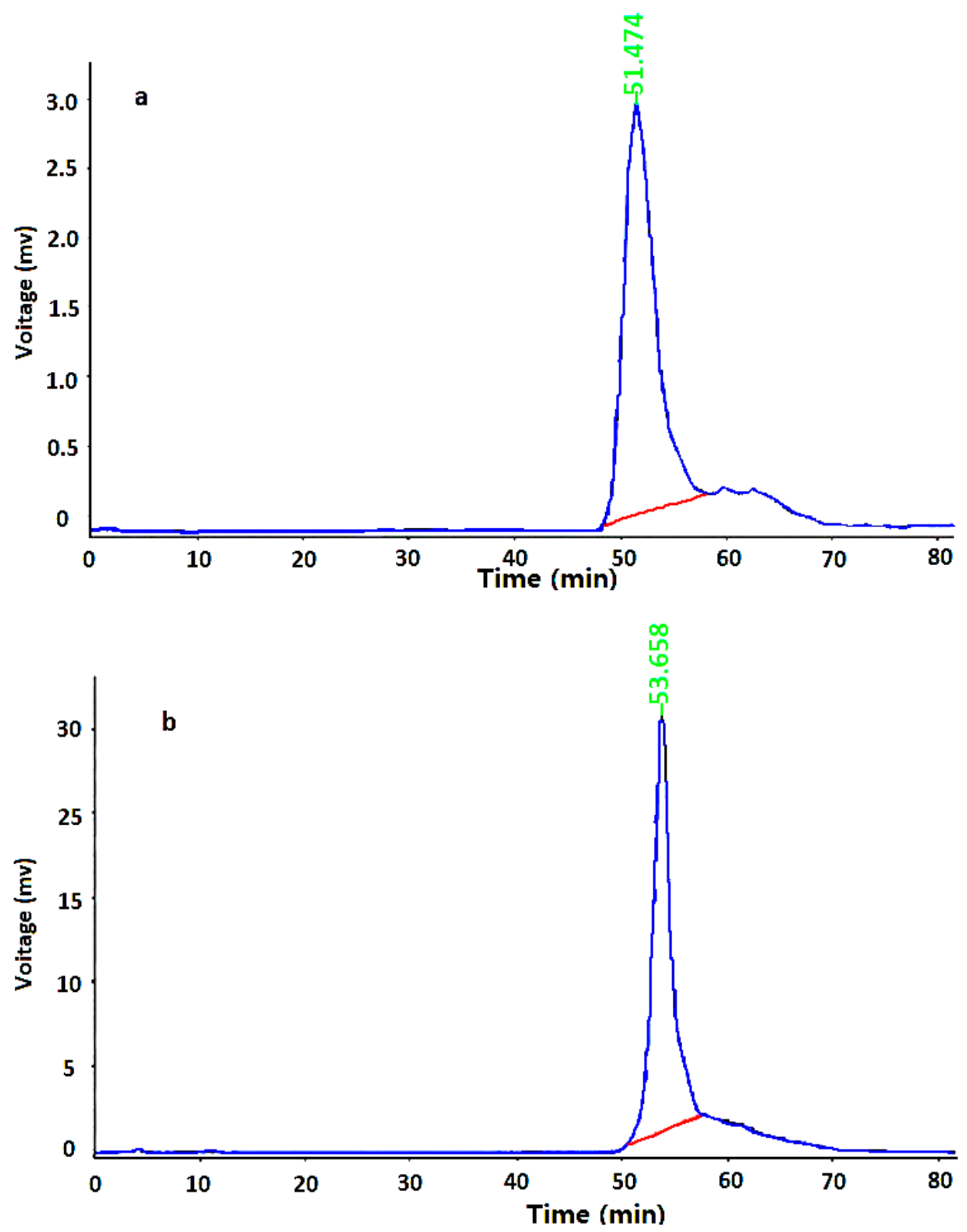
2.2.2. Ultraviolet (UV) and Fourier Translation Infrared (FT-IR) Spectrometric Characterization of PEA

The UV spectra of PEA are shown in Figure 3. No significant absorption at 260-280 nm was observed in the UV spectrum of PEA-1 and PEA-2, indicating the presence of impossible protein.

An FT-IR spectroscopy is used to investigate the vibrations of molecules and polar bonds between the different atoms. It is possible to analyze the structures of polysaccharides, such as monosaccharide types, glucosidic bonds, and functional groups, using an FT-IR spectroscopy. PEA-1 and PEA-2 were characterized by FT-IR spectroscopy (Figure 4). A strong and broad absorption peak at $3400 \mathrm{~cm}^{-1}$ for O-H stretching vibrations, a peak at $2930 \mathrm{~cm}^{-1}$ for $\mathrm{C}-\mathrm{H}$ stretching vibrations, and a strong extensive absorption in the region of $900-1200 \mathrm{~cm}^{-1}$ for coupled $\mathrm{C}-\mathrm{O}$ and $\mathrm{C}-\mathrm{C}$ stretching and $\mathrm{C}-\mathrm{OH}$ bending vibrations were observed in crude PEA-1 and PEA-2, indicating the characteristic absorptions of polysaccharides.

Figure 3. The UV spectra of (a) PEA-1 and (b) PEA-2.
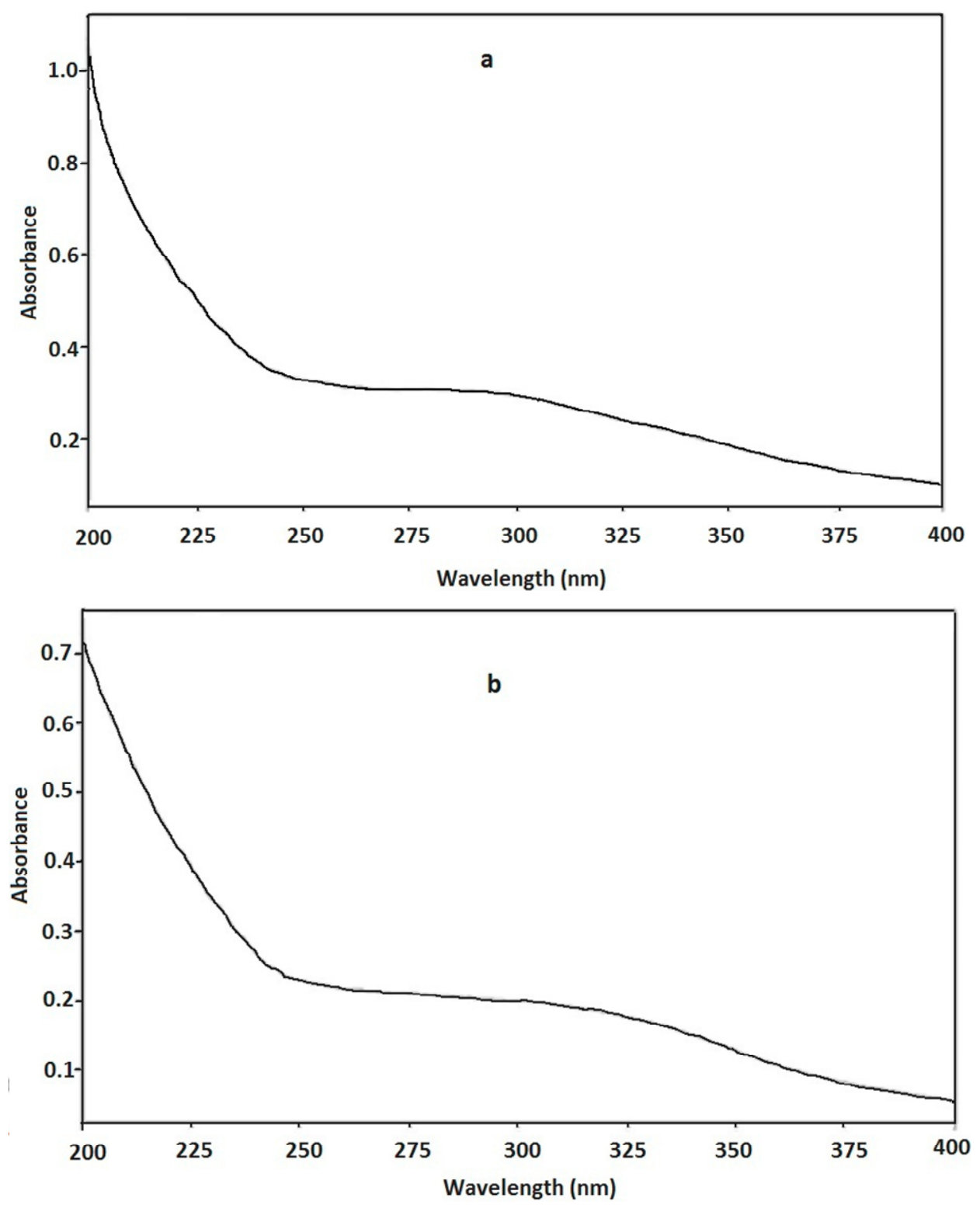
Figure 4. IR spectrum of (a) PEA-1and (b) PEA-2.
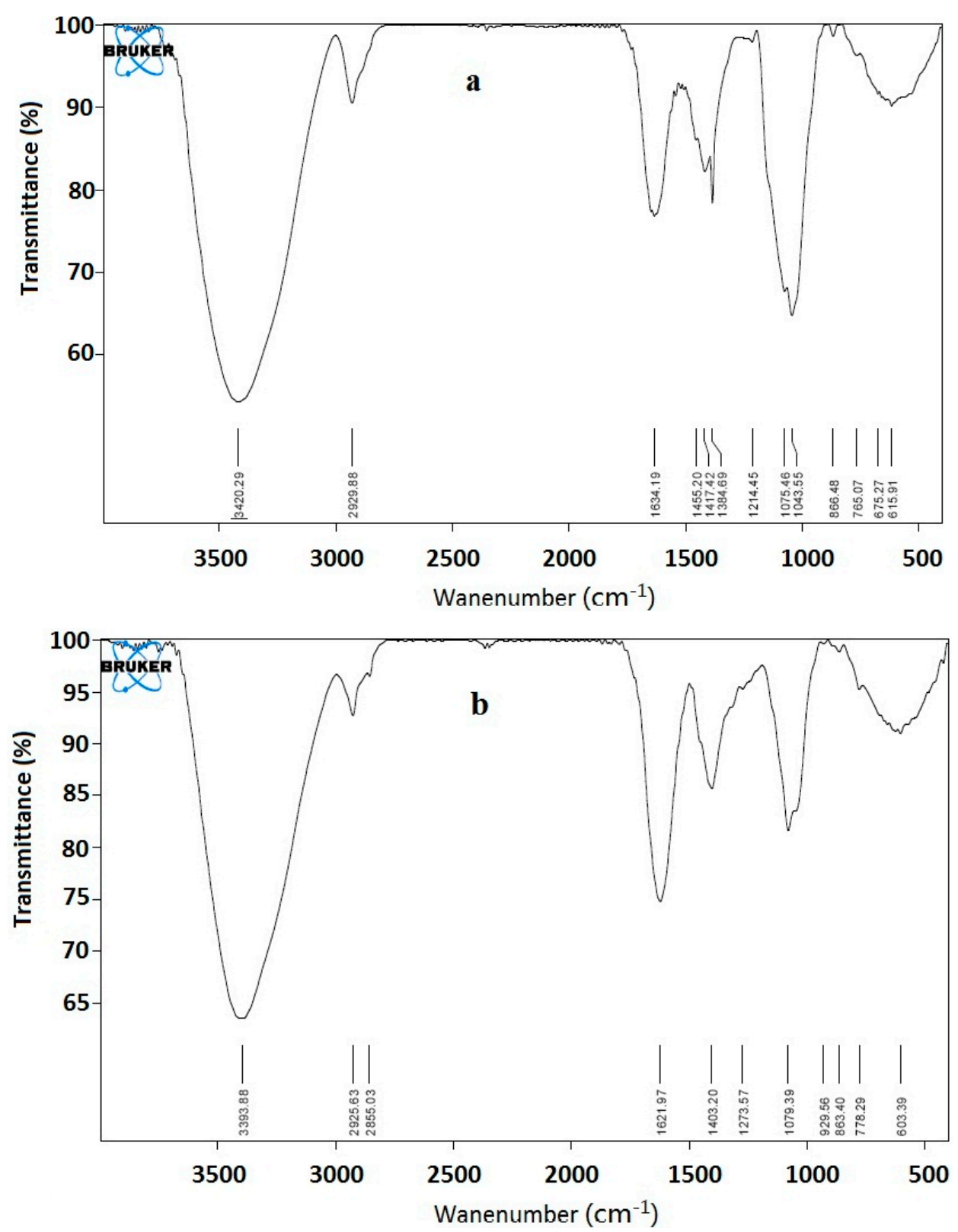

\subsubsection{Sugar Compositions of PEA-1 and PEA-2}

The monosaccharide composition of PEA-1 and PEA-2 were determined by gas chromatograph-mass spectrometer (GC-MS) and compared with the monosaccharide standards. The result showed that PEA-1 was mainly composed of rhamnose, xylose, mannose, glucose, and galactose, respectively, and the component of PEA-2 were rhamnose, mannose, glucose, and galactoserespectively (Table 1).

Table 1. Components of monosaccharide of polysaccharides.

\begin{tabular}{ccccccc}
\hline \multirow{2}{*}{ Sample } & \multicolumn{3}{c}{ Monosaccharide Composition (Relatively Mass \%) } & Polysaccharide \\
\cline { 2 - 5 } & Rha. & Xyl. & Man. & Glu. & Gal. & Content (\%) \\
\hline PEA-1 & 37.1 & 1.6 & 6.3 & 35.4 & 19.6 & 98.8 \\
PEA-2 & 12.4 & ND $^{\text {a }}$ & 7.1 & 42.4 & 38.1 & 97.3 \\
\hline
\end{tabular}

ND ${ }^{\mathrm{a}}$ : not detected. 


\subsection{Antioxidant Activity}

2.3.1. Effect of Scavenging 1,1-Diphenyl-2-picryl-hydrazyl (DPPH) Radicals

1,1-Diphenyl-2-picryl-hydrazyl (DPPH) assay is based on the reduction of DPPH radicals in the presence of a hydrogen donating antioxidant, resulting in the formation of a stable non-radical form DPPH-H and color fades ups. As shown in Figure 5, PEA-1 and PEA-2 showed obvious scavenging effect on DPPH radical in a dose dependent manner. At the concentrations ranged from 0.1 to $0.5 \mathrm{mg} / \mathrm{mL}$, the scavenging ability on DPPH radical of PEA-1 was much higher than PEA-2. At the concentration of $0.5 \mathrm{mg} / \mathrm{mL}$, PEA-1 possessed strong free radical scavenging effects of DPPH radicals (scavenging activity $88.1 \%$ ). The $\mathrm{IC}_{50}$ values of scavenging DPPH radicals were 0.27 and $0.38 \mathrm{mg} / \mathrm{mL}$ for PEA-1 and PEA-2.

Figure 5. DPPH radical scavenging activities and hydroxyl radical scavenging activities of PEA-1, PEA-2 and Vitamin C (Vc). Each value is the Mean \pm standard deviation (SD) of triplicate measurements.
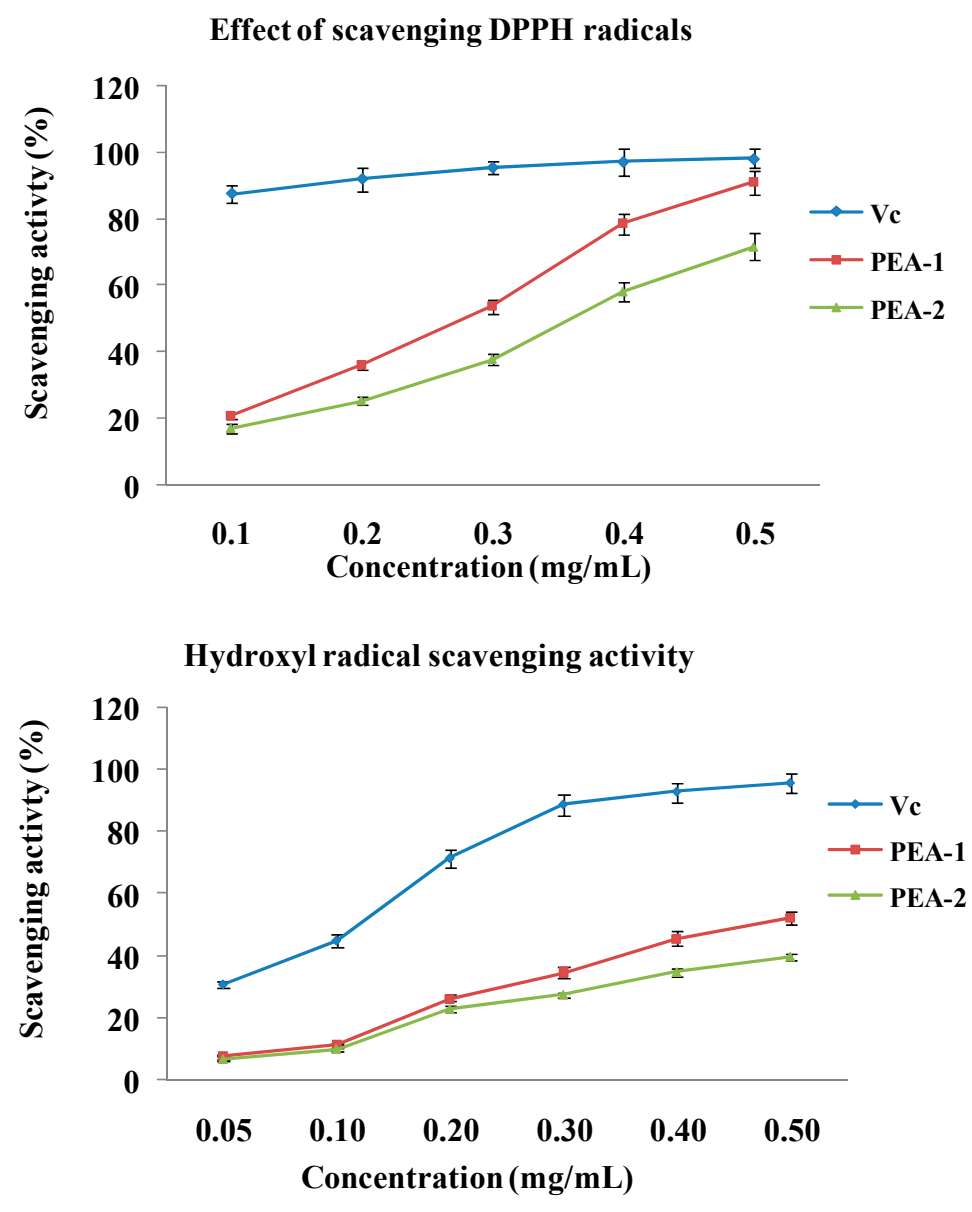

\subsubsection{Measurement of Hydroxyl Radical Scavenging Activity}

The hydroxyl radical is considered to be mainly responsible for oxidative injury of biomolecules. As shown in Figure 5, all samples exhibited obvious scavenging activity on hydroxyl radical in a dose dependent pattern at all concentrations. PEA-1 had the stronger activity than PEA-2. At the $0.5 \mathrm{mg} / \mathrm{mL}$, the scavenging activities of PEA-1 and PEA-2 were $52.3 \%$ and $39.8 \%$, respectively. The 
scavenging hydroxyl radical activity was attributed to various mechanisms, such as suppression against hydroxyl radical generation, decomposition of peroxides, prevention of continued hydrogen abstraction, and radical scavenging. The possibility of scavenging hydroxyl radical by polysaccharide, was that polysaccharide could combine with radical and terminate the radical chain reaction [11].

PEA-1 and PEA-2 have different structures and monosaccharide compositions, which lead to the antioxidant difference between PEA-1 and PEA-2. PEA-1 is mainly composed of rhamnose (37.1\%), glucose $(35.4 \%)$, and galactose (19.6\%); the component of PEA-2 major are rhamnose $(12.4 \%)$, glucose $(42.4 \%)$, and galactose (38.1\%). The major difference is the different content of rhamnose and galactose between PEA-1 and PEA-2. But, the absolute configuration of polysaccharide is difficult to confirm. So, the antioxidant difference between PEA-1 and PEA-2 is hard to explain clearly.

\section{Experimental Section}

\subsection{Materials and Supplies}

Elaeagnus angustifolia L. was purchased from local market (Shihezi, Xijiang, China). The pulp was separated manually from the fruit. Then powdered, and kept at room temperature for study.

Monosaccharides (D-glucose, L-rhamnose, D-xylose, L-arabinose, L-mannose, L-fucose, D-galactose) were purchased from Sigma-Aldrich Co. (Saint Louis, MO, USA). Trifluoroacetic acid, DEAE-Cellulose, Sephadex G-100, 1-diphenyl-2-picryl-hydrazyl, and other chemicals were obtained from New Sanli chemical reagents company (Xi'an, Shaanxi, China.).

\subsection{Isolation and Purification of the Extracellular Polysaccharide}

The powder of Elaeagnus angustifolia L. pulp was extracted with water at $80{ }^{\circ} \mathrm{C}$. Then the extraction was filtered through cheesecloth. The liquids were concentrated under reduced pressure at $40{ }^{\circ} \mathrm{C}$, and four-fold volume of $95 \%(v / v)$ ethanol was added. The resulting precipitate was recovered by using centrifugation at $3500 \mathrm{rpm}$ for $10 \mathrm{~min}$, and dialyzed in cellulose membrane (molecular weight cut-off 3500) against flowing distilled water for $72 \mathrm{~h}$. The retained fraction was recovered, concentrated under reduced pressure, and freeze-dried. The solution of crude polysaccharide was mixed with Sevage reagents (chloroform-butanol 4:1, v/v), based on certain percentage under magnetic stirring for $20 \mathrm{~min}$, to remove protein at the liquid-junction. Then, the crude polysaccharide was fractionated on DEAE-Cellulose 52 column (Beijing Huamaike Biotechnology Co., LTD, Beijing, China) elution with step-wise gradient of $0,0.1$ and $0.3 \mathrm{~mol} / \mathrm{L}$ ( $\mathrm{NaCl}$ solution). The total sugar content of the fractions was determined by the phenol-sulfuric acid method. Subfractions eluted with water, were pooled, dialyzed, and further purified on Sephadex G-100 column (Shanghai Haoran Biological Technology Co., LTD, Shanghai, China) with water as eluent. The major subfractions (PEA-1 and PEA-2) were pooled and freeze-dried.

\subsection{Determination of Total Polysaccharide Yield}

The polysaccharide content was measured by phenol-sulfuric method using D-glucose as a standard. The yield of crude polysaccharide was calculated as a percentage of the weight of the dry sample weight. The percentage total polysaccharide yield (\%) is calculated as follows: 


$$
\text { Total polysaccharide yield }(\%)=\frac{\text { Total polysaccharide weight }}{\text { Dry sample weight }} \times 100 \%
$$

\subsection{Preliminary Characterization of PEA-1 and PEA-2}

\subsubsection{Determination of Molecular Weights}

The molecular weight determination of PEA was performed using size-exclusion HPLC chromatography. All samples $(10.0 \mathrm{mg})$ were dissolved in distilled water $(1.0 \mathrm{~mL})$, passed through a $0.45 \mu \mathrm{m}$ filter, and applied to a gel-filtration chromatographic column. The column was maintained at a temperature of $25{ }^{\circ} \mathrm{C}$ and eluted with $0.1 \mathrm{M} \mathrm{Na}_{2} \mathrm{SO}_{4}$ solution in PBS buffer $(0.01 \mathrm{M}, \mathrm{pH}$ 6.8) at a flow rate of $0.8 \mathrm{~mL} / \mathrm{min}$.

\subsubsection{UV and FT-IR Spectrometric Analysis}

UV spectrum of PEA was recorded with a UV-2450 spectrophotometer (Shimadzu, Kyoto, Japan). FT-IR spectrum of PEA was recorded with a FT-IR spectrometer (Tensor 27, Baruker, Bremen, Germany) using the $\mathrm{KBr}$ disks method. Briefly, samples were dried at $35-44{ }^{\circ} \mathrm{C}$ in vacuum over $\mathrm{P}_{2} \mathrm{O}_{5}$ for $48 \mathrm{~h}$, ground with potassium bromide $(\mathrm{KBr})$ powder, and then pressed into pellets for FT-IR spectral measurement in the frequency range of $4000-400 \mathrm{~cm}^{-1}$.

\subsubsection{Analysis of Monosaccharide Composition}

The compositional analysis of PEA-1 and PEA-2 were performed by the alditol acetate method [12], with minor modifications. Firstly, the polysaccharide was hydrolyzed by $2 \mathrm{M}$ trifluoroacetic acid at $121{ }^{\circ} \mathrm{C}$ for $3.5 \mathrm{~h}$, followed by reduction in distilled water with $\mathrm{NaBH}_{4} 1.5 \mathrm{~h}$ at room temperature. Secondly, hydrolysate was acetylated by acetic anhydride with pyridine as the catalyst at $100{ }^{\circ} \mathrm{C}$ for $1 \mathrm{~h}$. Finally, the alditol acetate derivatives produced were separated by gas chromatography (GC) (7890A-G3440A, Agilent, Palo Alto, CA, USA).

\subsection{Determination of Antioxidant Activities of PEA-1 and PEA-2 in Vitro}

\subsubsection{Effect of Scavenging DPPH・ Radicals}

The scavenging activity on DPPH free radical (DPPH•) was measured as described previously [13-15], with slight modification. Briefly, $0.1 \mathrm{mM}$ solution of $\mathrm{DPPH} \bullet$ in methanol was prepared and $1.0 \mathrm{~mL}$ of this solution was added to $3.0 \mathrm{~mL}$ of the polysaccharides of various concentrations $(0.1,0.2,0.3,0.4$, and $0.5 \mathrm{mg} / \mathrm{mL}$ ) in water. The mixture was shaken and incubated at $25{ }^{\circ} \mathrm{C}$ for $30 \mathrm{~min}$ in the dark. Then the absorbance was measured at $517 \mathrm{~nm}$. Vitamin $\mathrm{C}(\mathrm{Vc})$ was used as the positive control.

\subsubsection{Assay of Hydroxyl Radical $(\bullet \mathrm{OH})$ Scavenging Activity}

The hydroxyl radical $(\bullet \mathrm{OH})$ scavenging activity was measured according to Fenton's reaction $[13,16,17]$. The hydroxyl radical was generated in a mixture of $1.0 \mathrm{~mL}$ of $5 \mathrm{mM} \mathrm{1,10-phenanthroline,} 1.0 \mathrm{~mL}$ of $0.05 \mathrm{M}$ sodium phosphate buffer (pH 7.4), $0.5 \mathrm{~mL}$ of $7.5 \mathrm{mM} \mathrm{FeSO}_{4}$ and $0.5 \mathrm{~mL}$ of $\mathrm{H}_{2} \mathrm{O}_{2}(3 \%, v / v)$. 
After addition of $2.0 \mathrm{~mL}$ sample solution, the mixture was incubated at $37^{\circ} \mathrm{C}$ for $1 \mathrm{~h}$. The absorbance was measured at $510 \mathrm{~nm}$. Deionized water and Vc were used as the blank and positive control, respectively.

\subsection{Statistical analysis}

All data were expressed as mean \pm standard deviation (SD).

\section{Conclusions}

In the present study, the preliminary characterization, antioxidants in vitro of polysaccharides from Elaeagnus angustifolia L. were investigated. Preliminary structural analysis indicated that PEA-1 and PEA-2 were mainly composed of rhamnose, glucose and galactose etc., respectively, and the average molecular weights for PEA-1 and PEA-2 were 9113 and 5020 Da, respectively. Antioxidant activities in vitro demonstrated that PEA-1 and PEA-2 had moderate scavenging activity and lipid peroxidation inhibition effect. These results suggested that PEA-1 and PEA-2 had potent antioxidant activities.

In order to develop and use it effectively, we will work on the animal-testing, which be used to evaluate the food safety and application potential.

\section{Acknowledgments}

Financial support from the National Natural Science Foundation of China (Grant No. 31270388), "Chun Sun Plan" of Shaanxi Province and the Fundamental Research Funds for the Central Universities (No. QN2011066) is gratefully acknowledged.

\section{Author Contributions}

Qingqing Chen completed the extraction and purification of polysaccharides; Juncheng Chen did the preliminary characterization of PEA-1 and PEA-2; Hongtao Du accomplished antioxidant activity of two polysaccharides; Qi Li and Jun Chen collected samples; Gechao Zhang pretreated samples; Hong Liu guided student to extract and separate polysaccharides; and Junru Wang instructed workers to research the preliminary characterization and antioxidant activity of two polysaccharides.

\section{Conflicts of Interest}

The authors declare no conflict of interest.

\section{References}

1. Huang, J.; Maimaiti, J.; Yang, C.; Wang, C. Present situation and prospect about the study of Elaeagnus angustifolia L. Chin. Wild Plant Resour. 2005, 24, 26-28.

2. Mirhydar, H. Encyclopedia of plants: Indications of plants in the prevention and treatment of diseases. Islamic Farhang Tehran. 1998, 2, 163-164.

3. Gürbüz, I.L.; Üstün, O.; Yesilada, E.; Sezik, E.; Kutsal, O. Anti-ulcerogenic activity of some plants used as folk remedy in Turkey. J. Ethnopharmacol. 2003, 88, 93-97. 
4. Ganslmayer, M.; Spertini, F.M.; Terrien, H.; Leimgruber, A.; Rahm, F.; Mosimann, B. Evaluation of acoustic rhinometry in a nasal provocation test with allergen. Allergy 1999, 54, 974-979.

5. Nishino, C.; Enoki, N.; Tawata, S.; Mori, A.; Kobayashi, K.; Fukushima, M. Antibacterial activity of flavonoids against Staphylococcus epidermidis, a skin bacterium. Agric. Biol. Chem. 1987, 51, 139-143.

6. Hosseinzadeh, H.; Ramezani, M.; Namjo, N. Muscle relaxant activity of Elaeagnus angustifolia L. fruit seeds in mice. J. Ethnopharmacol. 2003, 84, 275-278.

7. Ramezani, M.; Hosseinzadeh, H.; Daneshmand, N. Antinociceptive effect of Elaeagnus angustifolia fruit seeds in mice. Fitoterapia 2001, 72, 255-262.

8. Ding, Y.; Wng, Z.; Ma, R.; Feng, G.; Xu, S.; Lu, F.; Tang, M.; Zheng, R. Anti-fatigue effect and mechanism of polysaccharides from Elaeagnus angustifolia L. Food Sci. 2010, 31, 255-257.

9. Zhao, H.; Zhang, G.; Tang, H.; Tian, L.; Wang, B. The assessment of assisting irradiation hazard protection function of Elaeagnus angustifolia polysaccharide on mice. Chin. Prev. Med. 2012, 13, $107-108$.

10. Lian, Y.; Chen, H.; Li, B.; Yang, J.; Yuan, J. Immunoloregulation effect of Elaeagnus angustifolia L: Polysaccharides in immunosuppressive mice. J. Anhui Agric. Sci. 2009, 37, 7481-7482.

11. Chen, R.; Liu, Z.; Zhao, J.; Chen, R.; Meng, F.; Zhang, M.; Ge, W. Antioxidant and immunobiological activity of water-soluble polysaccharide fractions purified from Acanthopanax senticosu. Food Chem. 2011, 127, 434-440.

12. Crowell, E.P.; Burnett, B.B. Determination of the carbohydrate composition of wood pulps by gas chromatography of the alditol acetates. Anal. Chem. 1967, 39, 5.

13. Cheng, H.; Feng, S.; Jia, X.; Li, Q.; Zhou, Y.; Ding, C. Structural characterization and antioxidant activities of polysaccharides extracted from Epimedium acuminatum. Carbohydr. Polym. 2013, 92, 63-68.

14. Jiang, C.; Xiong, Q.; Gan, D.; Jiao, Y.; Liu, J.; Ma, L.; Zeng, X. Antioxidant activity and potential hepatoprotective effect of polysaccharides from Cyclina sinensis. Carbohydr. Polym. 2013, 91, 262-268.

15. Wu, C.; Wang, X.; Wang, H.; Shen, B.; He, X.; Gu, W.; Wu, Q. Extraction optimization, isolation, preliminary structural characterization and antioxidant activities of the cell wall polysaccharides in the petioles and pedicels of Chinese herbal medicine (Qian Euryale ferox Salisb.). Int. J. Biol. Macromol. 2014, 64, 458-467.

16. Cheng, H.; Feng, S.; Shen, S.; Zhang, L.; Yang, R.; Zhou, Y.; Ding, C. Extraction, antioxidant and antimicrobial activities of Epimedium acuminatum Franch. polysaccharide. Carbohydr. Polym. 2013, 96, 101-108.

17. Wang, X.; Zhang, Z.; Yao, Q.; Zhao, M.; Qi, H. Phosphorylation of low-molecular-weight polysaccharide from Enteromorpha linza with antioxidant activity. Carbohydr. Polym. 2013, 96, 371-375.

(C) 2014 by the authors; licensee MDPI, Basel, Switzerland. This article is an open access article distributed under the terms and conditions of the Creative Commons Attribution license (http://creativecommons.org/licenses/by/3.0/). 